\title{
Integration of microorganism Vorticella convallaria and poly (ethylene glycol) diacrylate hydrogel for biohybrid systems
}

\author{
Moeto NAGAI*, Kei KATO* and Takayuki SHIBATA* \\ * Department of Mechanical Engineering, Toyohashi University of Technology \\ 1-1 Hibarigaoka, Tenpaku-cho, Toyohashi, Aichi 444-8580, Japan \\ E-mail: nagai@me.tut.ac.jp
}

Received 24 July 2016

\begin{abstract}
Biohybrid systems, constructed by combining biological cells and artificial components, have the potential to resolve the current technical limits of traditional microactuators. The aim of this study is to bring poly(ethylene glycol) diacrylate (PEG-DA) movable components, formed by microfluidic in situ photolithography, into biohybrid Vorticella-based systems. This paper reports conditions for integrating PEG-DA hydrogels and actuators of Vorticella convallaria ( . convallaria). PEG-DA hydrogels were formed in a polydimethylsiloxane (PDMS) microfluidic channel by in-situ photolithography techniques. Basic photopolymerization properties of PEG-DA hydrogels in a PDMS microchannel were studied by changing the magnification of an objective lens and light intensity. To incorporate a PEG-DA structure with $V$. convallaria, we studied two possible integration methods: 1) hydrogel formation after cell placement and 2) cell placement after hydrogel formation. To examine the integration methods, we evaluated the viability of $V$. convallaria in a PEG-DA solution mixed with photoinitiators or in a buffer after the formation of a PEG-DA hydrogel. A viability assay in a PEG-DA solution revealed that lower concentrations of PEG-DA and photoinitiator improved cellular viability. To establish the second integration method, we designed and fabricated a microfluidic device to transport a $V$. convallaria cell to a microchamber containing a polymerized PEG-DA structure. Cell viability in a microchamber was evaluated after replacing a PEG-DA solution with a culture medium. Vorticella was active and generated a flow, which was characterized by particle image velocimetry (PIV). A higher molecular weight PEG-DA increased $V$. convallaria viability. The conditions for forming a hydrogel structure while maintaining Vorticella activity were clarified as a method for incorporating a movable component into biohybrid systems.
\end{abstract}

Key words: Biohybrid system, In situ photolithography, PEG-DA, Hydrogel formation, Microfluidic device, Vorticella convallaria, Bioactuator, Viability assay, Movable component

\section{Introduction}

Engineering systems have been miniaturized based on microfabrication technologies (Madou, 2011). One successful example is found in biochemical analysis systems, which have been miniaturized and used as lab-on-a-chip devices (Neužil, et al., 2012). Dynamic functions of such microsystems depend on microactuators, which are composed of artificial mechanical components and require external power sources such as electrical (Toonder, et al., 2008) and magnetic (Lu, et al., 2002) sources. These actuation principles have limitations in the miniaturization of the entire systems. These problems are supposed to be resolved by a new element that differs in principle.

A biohybrid system, which assembles biological cells and artificial components, is expected to provide a solution to the problem (Akiyama, et al., 2013, Carlsen and Sitti, 2014). Biological cells convert chemical energy into the mechanical work of motors; they can remove external circuits for an energy supply. The integration of biological cells in microsystems enables us to obtain the advantages of cells and artificial structures, which affords better functionality compared to that of conventional engineered systems. We have focused on applications of the motile microorganism, Vorticella convallaria (V. convallaria) (Nagai, et al., 2010, Nagai, et al., 2013a). V. convallaria performs two major types of motions: (1) Cilia of $V$. convallaria generate a flow surrounding the oral part of the cell body; (2) A stalk of $V$. 
convallaria exhibits repeating extension-and-contraction motions. These biological motors have potential as actuators in microsystems. In earlier studies, we placed cells of $V$. convallaria in a static flow channel structure and presented mixing of solutions on a micro scale.

Movable components in a static structure enable us to achieve the dynamic change of biohybrid Vorticella-based systems. It is essential to find a method of fabricating movable components, which allows the dynamic state change of a microsystem. We found microfluidic in situ photolithography technique (Dendukuri, et al., 2006, Chung, et al., 2008) can offer not only fabrication of a movable micro-structure but also new polymeric materials and rapid prototyping in a microchannel. This technology projects UV light to photocurable resin in a channel, which forms a microstructure and provides dynamic formation of a patterned structure. Poly(ethylene glycol) diacrylate (PEG-DA) is commonly used as a monomer for optofluidic lithography because of its biocompatibility.

Conditions for forming a PEG-DA structure while keeping Vorticella active should be elucidated to develop biohybrid Vorticella-based systems incorporating PEG-DA movable parts. Mammalian cells have been encapsulated in PEG-DA hydrogels (Koh, et al., 2003, Koh and Pishko, 2006) and cultured on PEG-DA microwells (Revzin, et al., 2003, Moeller, et al., 2008). However, biocompatibility of PEG-DA is dependent on organism species. Methods for integrating the ciliated protozoa, Vorticella, into a PEG-DA micro-structure have not been clarified.

We investigated conditions for integrating a PEG-DA structure and a $V$. convallaria cell to introduce microfluidic in situ photolithography techniques to biohybrid systems. We characterized basic properties for forming a PEG-DA structure in a PDMS microchannel using an optical setup. To incorporate a PEG-DA structure with $V$. convallaria, we studied two possible integration methods: 1 ) formation of a PEG-DA structure in the presence of $V$. convallaria in a PEG-DA solution and 2) formation of a PEG-DA structure in a chamber without $V$. convallaria followed by transportation of a cell to the chamber. To examine the first method, viability of $V$. convallaria was evaluated in a PEG-DA solution. To establish the second method, a $V$. convallaria cell was transported through designed microchannels and into a microchamber by flow. The viability of a cell in the microchamber was measured after replacing the PEG-DA solution with culture medium. $V$. convallaria was combined with a PEG-DA structure in a chamber; a flow field, generated by $V$. convallaria, was characterized by PIV.

\section{Experimental methods}

\subsection{Optical and measurement setup}

Figure 1(a) shows the experimental setup for ultraviolet photo patterning by optofluidic lithography. The optical setup was based on an inverted microscope (Eclipse TE2000-U, Nikon). A 100-W mercury lamp (C-SHG1, Nikon) was used as a source of UV light. A PEG-DA solution was mixed with a photoinitiator and filled in a microfluidic channel. We selectively irradiated UV light (330-380 nm) to the solution using a filter cube (UV-2A, Nikon). A neutral-density (ND) filter was used to adjust the intensity of the light. We inserted a photomask containing a pattern in the field-stop of the microscope. This optical plane was conjugated to the substrate on the microscope stage. The mask pattern was reduced and projected through an objective lens. The patterning place was scanned with a microscope stage.

Polymerized PEG-DA hydrogels and the motion of $V$. convallaria were visualized with the inverted microscope. A movie was recorded with a CCD camera (DIGITAL SIGHT DS-2Mv, Nikon) at $5 \mathrm{fps}$. We characterized the fundamental performance of the PEG-DA micro-structure with this optical setup.

\subsection{Preparation of PEG-DA solution and Vorticella convallaria}
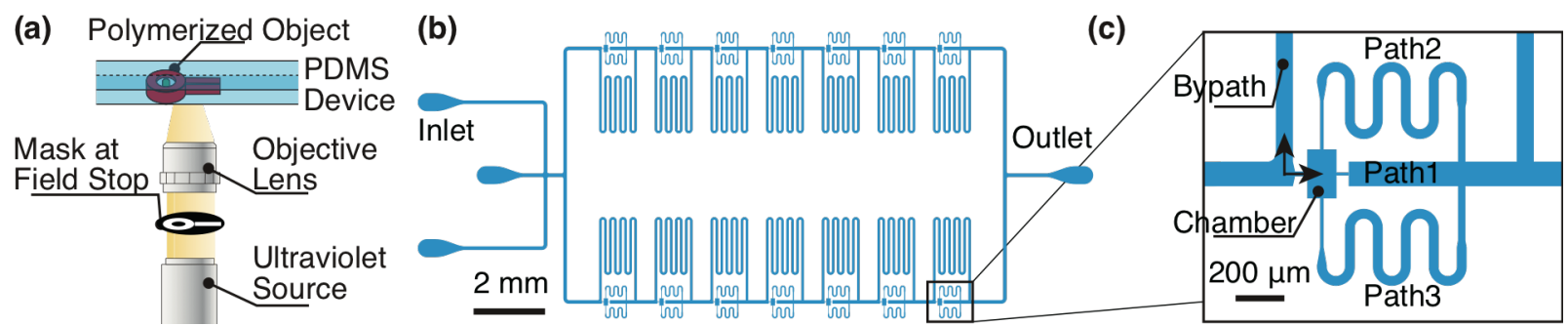

Fig. 1 Schematic of experimental setup for integration of $V$. convallaria and a PEG-DA micro-structure. (a) Optical setup for polymerization of a PEG-DA solution in a microfluidic device. (b), (c) Layout of a microchannel for cell trapping. (b) Whole channel composed of $7 \times 2$ chambers. (c) Enlarged view of 1 microchamber. 
We used PEG-DA (Mn 250 or 700, Sigma-Aldrich) as the monomer and Irgacure 651 (2,2-dimethoxy-2phenylacetophenone [DMPA], Tokyo Chemical Industry) as the photoinitiator. We prepared a polymer precursor mixture from a $5 \mathrm{wt} \%$ solution of DMPA in PEG-DA. This precursor solution was exposed to UV light to form a PEG-DA hydrogel.

A cell suspension of $V$. convallaria was prepared according to a previous method (Vacchiano, et al., 1991, Nagai, et al., 2013b). It was extracted from $1-\mathrm{g} / \mathrm{L}$ of wheat grass powder (Pines Wheat Grass, Pines International, Inc.) by autoclaving at $121^{\circ} \mathrm{C}$ and removing the solid content by filtration. The cells were incubated in a $1-\mathrm{L}$ flask at $20^{\circ} \mathrm{C}$ while shaking for $24 \mathrm{~h}$ to obtain floating $V$. convallaria cells.

\subsection{Fabrication of two types of microfluidic devices}

We fabricated two types of microfluidic devices in polydimethylsiloxane (PDMS) by soft lithography. The first type was a single layer device, composed of straight channels with a $10 \mathrm{~mm}$-length and $300 \mu \mathrm{m}$-width. This device was used for hydrogel polymerization experiments in the absence of $V$. convallaria. The second type was a double layer device and used for 2) formation of a PEG-DA structure in a microchamber without $V$. convallaria and transportation of a cell to the chamber (Figs. 1b, c).

The second device patterned $V$. convallaria in microchambers by fluidic forces based on the calculation of fluidic resistances. This working principle was described elsewhere (Tan and Takeuchi, 2007, Oh, et al., 2012). The flow resistances of channels were designed to trap a cell in a chamber (Fig. 1c). The rate of flow to the chamber was stronger than one through a bypath channel before trapping a cell inside the chamber. The flow transported the cell to the chamber. The cell became trapped in the chamber, then a new cell flowed through a bypath channel. One chip was composed of 14 chambers. The dimensions of each chamber were $200 \mu \mathrm{m}$ width $\times 120 \mu \mathrm{m}$ length, and $40 \mu \mathrm{m}$ height. The heights of paths $1-3$, in Fig. 1(c), were $\sim 10 \mu \mathrm{m}$, to prevent the escape of $V$. convallaria from the chamber. All other channels were $\sim 40 \mu \mathrm{m}$ high.

The two types of devices were fabricated by preparing two types of molds from thick negative photoresist SU-8 3050 (Microchem) on a 3-inch silicon wafer. SU-8 was dispensed to the silicon wafer and spin coated under two different conditions. (1) The first single layer mold was prepared, by spin coating SU-8 at 3,000 rpm for 60 s. (2) Preparation of the second double layer mold required spin coating the first SU-8 layer at 7,000 rpm for $60 \mathrm{~s}$, and the second layer at $4,000 \mathrm{rpm}$ for $60 \mathrm{~s}$. The resist was prebaked at $65^{\circ} \mathrm{C}$ and $95^{\circ} \mathrm{C}$, exposed through a photomask, and baked at $65^{\circ} \mathrm{C}$ and $95^{\circ} \mathrm{C}$. We developed the resist in 2-methoxy-1-methylethyl acetate. The molds were treated in the vapor of trichloro $(1 \mathrm{H}, 1 \mathrm{H}, 2 \mathrm{H}, 2 \mathrm{H}$-perfluorooctyl) silane (Sigma-Aldrich) for $2 \mathrm{~min}$ to further ease the demolding process.

A PDMS pre-polymer (Sylgard 184, Dow Corning Toray) was mixed at a 10:1 ratio of base to curing agent. The PDMS mixture was poured over the molds and degassed in a vacuum chamber. PDMS was cured at $80^{\circ} \mathrm{C}$ for $1 \mathrm{~h}$. The replicated PDMS chip was released from the mold. We punched $0.5 \mathrm{~mm}$ inlet and outlet holes in the chip. The PDMS chips were cleaned in deionized water (DIW).

The PDMS chip and a coverslip $(24 \mathrm{~mm} \times 36 \mathrm{~mm}, 0.12-0.17 \mathrm{~mm}$ thick (Matsunami Glass) were treated in air plasma with a plasma treater (YHS-R, Sakigake Semiconductor) for $30 \mathrm{~s}$. The PDMS chip and the coverslip were assembled and baked at $80^{\circ} \mathrm{C}$ for $15 \mathrm{~min}$ for permanent bonding. This bare glass substrate was critical for fixing a polymerized microstructure on the glass surface.

(a)

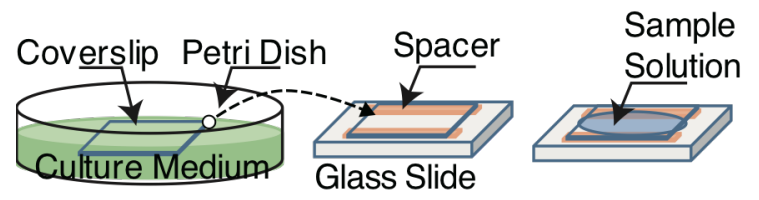

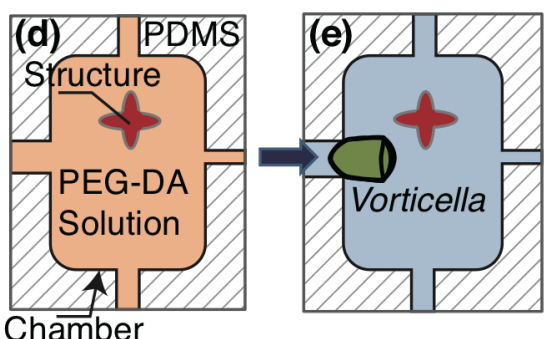

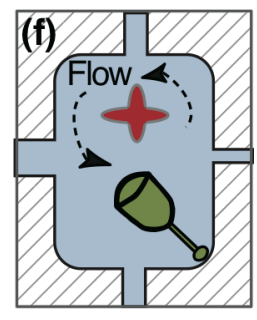

Fig. 2 Assessment of cellular viability in two conditions for studying the two possible integration methods. (a)-(c) First viability assay in a PEG-DA solution for examining the first integration method. (a) Culture of cells on a coverslip. (b) The coverslip and a glass slide building a chamber. (c) Injection of a PEG-DA solution to the chamber. (d)-(f) Second viability assay in a microchamber after replacing a PEG-DA solution with a culture medium for establishment of the second integration method. (d) Polymerization of a hydrogel structure inside a chamber. (e) Cell injection into the chamber containing the structure. (f) Flow generation by $V$. convallaria in the chamber. 


\subsection{Assessment of cellular viability in two conditions}

$V$. convallaria viability was assessed in two conditions to investigate the two possible integration methods. 1) In the first viability assay, $V$. convallaria was in a PEG-DA precursor solution. 2) The second assay measured V. convallaria viability in a microfluidic chamber after replacing a PEG-DA precursor solution with cell culture medium. Our criterion for assessing viability was that a cell had to show ciliary motion or contraction-extension.

1) The first viability assay used a stock solution, composed of $2 \mathrm{wt} \%$ Irgacure 2959 (I-2959; (2-hydroxy-1-(4(hydroxyetoxy)phenyl)-2methoxy-1-propanone, Tokyo Chemical Industry) in PEG-DA (Mn 700) (Figs. 2a-c). The stock solution was diluted with culture medium. The polymer precursor concentration was adjusted to 5, 7.5, and 10\% (v/v). A flow chamber was formed by placing two spacers between a coverslip and a glass slide containing adherent $V$. convallaria cells. The PEG-DA solution was injected into the flow chamber. The cellular motions in the solution were measured.

2) The second viability assay procedure measured the movement of $V$. convallaria cells injected into a microchamber, where a polymerized hydrogel was formed. Figures 2(d)-(f) show the flow process for integrating $V$. convallaria into the structure. A microchamber was filled with a PEG-DA solution (Mn 250 or 700) containing 5 wt $\%$ DMPA. The solution was exposed to UV light and formed a structure in the chamber (Fig. 2d). The PDMS chamber was washed with DIW. Water was continuously injected by hydrostatic pressure for $15 \mathrm{~h}$. The amount of total DIW for washing was $0.5 \mathrm{~mL} / 15$ h. A suspension of $V$. convallaria was introduced into the chip and trapped in the chamber (Fig. 2e). Cellular activity was measured after the formation of a structure and introduction of cells. The wheat grass powder extraction buffer served as a buffer.

\subsection{Measurement of activity and flow in microchamber containing Vorticella convallaria and hydrogel}

$V$. convallaria cells were trapped inside of microchambers and then incubated for establishment of the second integration method. We measured the activities of $V$. convallaria cells and observed a flow, which was generated by the ciliary motion of $V$. convallaria. Cells were introduced into chambers, after PEG-DA hydrogels had formed using a PEGDA (Mn 700) and DMPA $5 \mathrm{wt} \%$ solution to keep $V$. convallaria active. We used a $20 \times$ objective lens to irradiate UV light for less than $1 \mathrm{~s}$. The structures and the channels were washed with DIW for $5 \mathrm{~h}$, after the fabrication process was completed. Entrapped cells in a microchamber were incubated and allowed to adhere to the chamber surface. Active $V$. convallaria started to generate a flow inside a chamber (Fig. 2f).

The flow was visualized by injecting a solution containing fluorescent particles into the chamber. The fluorescent particles had a $0.5 \mu \mathrm{m}$ diameter (Fluoresbrite ${ }^{\circledR}$ YG Carboxylate Microspheres) and their adjusted concentration was $4.5 \times 10^{7}$ particles $/ \mu \mathrm{L}$. The observed results were evaluated by particle image velocimetry (PIV). The flow was captured with a fluorescent microscope (Ti-U, Nikon) equipped with a cooled CCD camera (DS-Qi1Mc, Nikon). The camera exposure time was set to $40 \mathrm{~ms}$ with a frame rate of $13.68 \mathrm{fps}$. To analyze a flow field based on PIV analysis, JPIV software was used with an interrogation window size of $20 \times 20$ pixels and a search window size of $32 \times 32$ pixels.

\section{Results and discussion}

\subsection{Characteristics of optofluidic lithography}
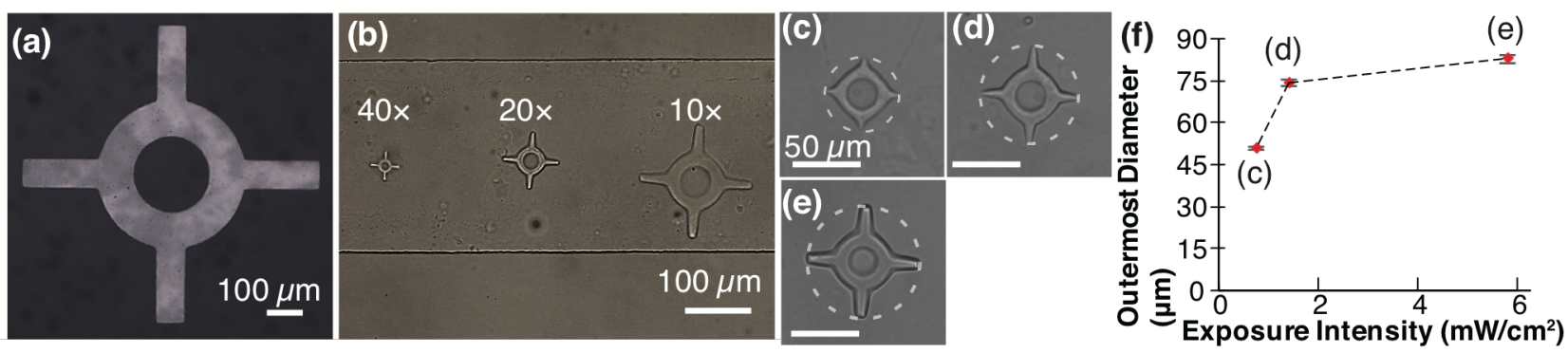

Fig. 3 Photopolymerization of a PEG-DA solution in a microfluidic channel. Micrographs of (a) a photomask and (b) polymerized objects in a PDMS channel through objective lenses at different magnification $(10 \times, 20 \times, 40 \times)$, irradiated for $2.0 \mathrm{~s}$ of UV exposure. DMPA (5 wt\%) in PEG-DA (Mn 250) was used as a polymer precursor. (c)-(e) PEG-DA structures at different intensities at (c) 0.75 , (d) 1.42 , or (e) $5.8 \mathrm{~mW} / \mathrm{cm}^{2}$. The dashed line shows the outermost diameters of the structures. (f) Outermost diameters of the polymerized objects at different intensities. 

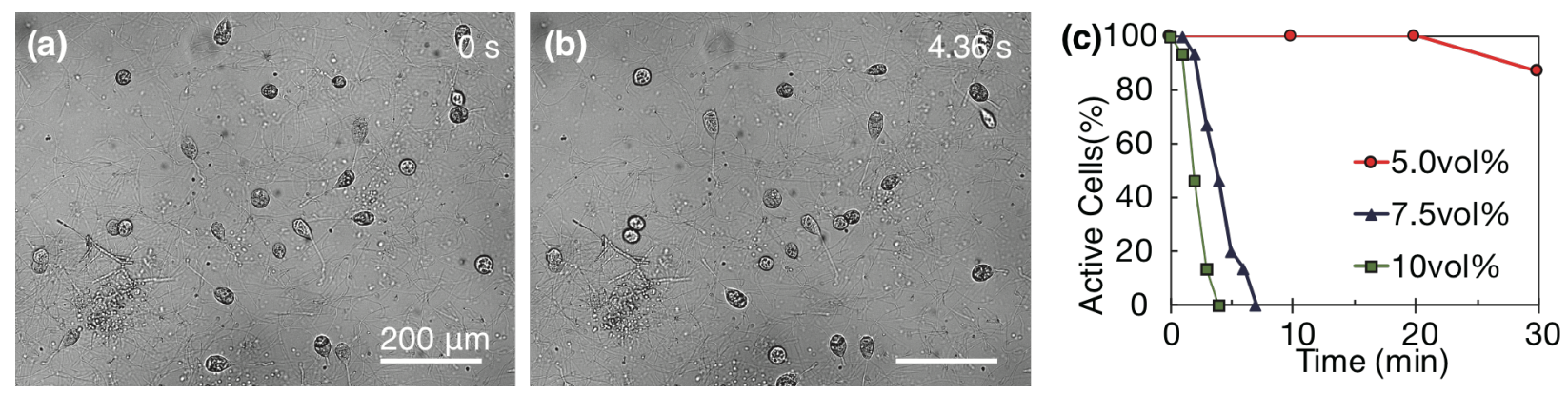

Fig. 4 Cellular viability assay in a PEG-DA solution (Mn 700) for verification of the first integration method. (a), (b) Microscope images of $V$. convallaria on a coverslip in a $5.0 \%(\mathrm{v} / \mathrm{v})$ solution. (c) Active cell ratio of $V$. convallaria in 5.0, 7.5, and 10\% (v/v) of PEG-DA precursor solutions, diluted with a culture medium.

UV was selectively irradiated to a liquid-phase photocurable hydrogel, and this irradiation formed a polymeric microstructure with designed features (Fig. 3). The dimensions of the channel, where the photocurable monomer was filled, were $300 \mu \mathrm{m}$ wide and $27.3 \pm 0.7 \mu \mathrm{m}$ (mean $\pm \mathrm{SD}, \mathrm{n}=4$ ) high. A gear-shape pattern with an inner diameter (ID) of 203 $\mu \mathrm{m}$ and outer diameter (OD) of $401 \mu \mathrm{m}$ of a central ring was contained in a photomask (Fig. 3a). This mask was used in combination with $10 \times, 20 \times$, and $40 \times$ magnification objective lenses to form hydrogel structures. We investigated reduction factors of the optical system for a fixed exposure time of $2 \mathrm{~s}$ (Fig. 3b). Three PEG-DA structures were fabricated in the same microchannel. ID and OD of polymerized objects were respectively reduced to $49 / 80 \mu \mathrm{m}$ at $10 \times, 25 / 39 \mu \mathrm{m}$ at $20 \times, 12 / 22 \mu \mathrm{m}$ at $40 \times$. These diameters corresponded to the reduction factors of $4.1 / 5.0$ (ID/OD) at $\times 10,8.1 / 10$ at $20 \times$, and $17 / 19$ at $40 \times$.

The intensity levels of UV light were changed with ND filters. We measured the effect on the polymerized diameter of a PEG-DA structure through a $20 \times$ objective (Figs. $3 \mathrm{c}-\mathrm{f}$ ). The exposure intensities were $0.75,1.42$, and $5.80 \mathrm{~mW} / \mathrm{cm}^{2}$. A central ring appeared in all conditions. Four pins around the ring were partially formed at $0.75 \mathrm{~mW} / \mathrm{cm}^{2}$ for $2 \mathrm{~s}$, then were fully formed at $5.80 \mathrm{~mW} / \mathrm{cm}^{2}$ for $2 \mathrm{~s}$. The outermost diameter of four pins increased as exposure intensity increased (Fig. 3f). These results indicate that it takes more than $10 \mathrm{~mJ} / \mathrm{cm}^{2}$ to completely form the structure, generated by polymerization of the prepared PEG-DA solution in the PDMS channel.

\subsection{Assessment of Vorticella convallaria viability in PEG-DA solution}

$V$. convallaria motion was observed in a flow chamber filled with PEG-DA precursor solutions (5.0, 7.5, and 10\% $(\mathrm{v} / \mathrm{v}))$ as the first viability assay described in 2.4. This viability assay was assessed to examine the first integration method. Figs. 4(a) and (b) show typical images of $V$. convallaria in the solution. At first, $V$. convallaria spontaneously repeated contraction and elongation in the solution. The cells decreased their activities with increasing time; they gradually stopped their contraction and extension motions.

We evaluated the percentage of active cells $(\mathrm{N}=15)$ in various concentrations of PEG-DA precursor solutions (Mn $700,5.0,7.5$, and $10 \%(\mathrm{v} / \mathrm{v}))$ for $30 \mathrm{~min}$. The activity of $V$. convallaria increased with decreasing PEG-DA concentrations (Fig. 4c). The percentage of active cells was highest in 5\% (v/v) solution. The active rate was $87 \%(=13 / 15)$ at $30 \mathrm{~min}$. Active rates decreased to $13 \%(=2 / 15)$ in $10 \%(\mathrm{v} / \mathrm{v})$ solution at $3 \mathrm{~min}$ and in $7.5 \%(\mathrm{v} / \mathrm{v})$ solution at $6 \mathrm{~min}$. This rapid decrease indicated that either PEG-DA or I-2959 was toxic to $V$. convallaria; the toxicity level was greater in $V$. convallaria compared to mammalian cells.

We have the possibility to perform the first integration method, which fabricates a PEG-DA structure formed in the presence of $V$. convallaria in a low concentration of a PEG-DA precursor solution. A PEG-DA and photoinitiator solution at a low concentration can be photo-patterned in an active cell environment. It was necessary to reduce the concentration of PEG-DA or I-2959 in order to keep $V$. convallaria active for a longer time; however, attention is required. The lower concentration of PEG-DA decreased the elastic modulus of hydrogel (Hahn, et al., 2007); the lower concentration of photoinitiators increased the crosslinking time (Mironi-Harpaz, et al., 2012). These relationships need to be considered to form a PEG-DA hydrogel around $V$. convallaria.

\subsection{Integration of Vorticella convallaria and a PEG-DA structure}

We fabricated a two-layer microfluidic device, which was used for establishment of the second integration method. The device had micro chambers with a height of $39.3 \pm 1.5 \mu \mathrm{m}(\mathrm{n}=3)$ and channels with a lower height of $11.0 \pm 0.3 \mu \mathrm{m}$ 

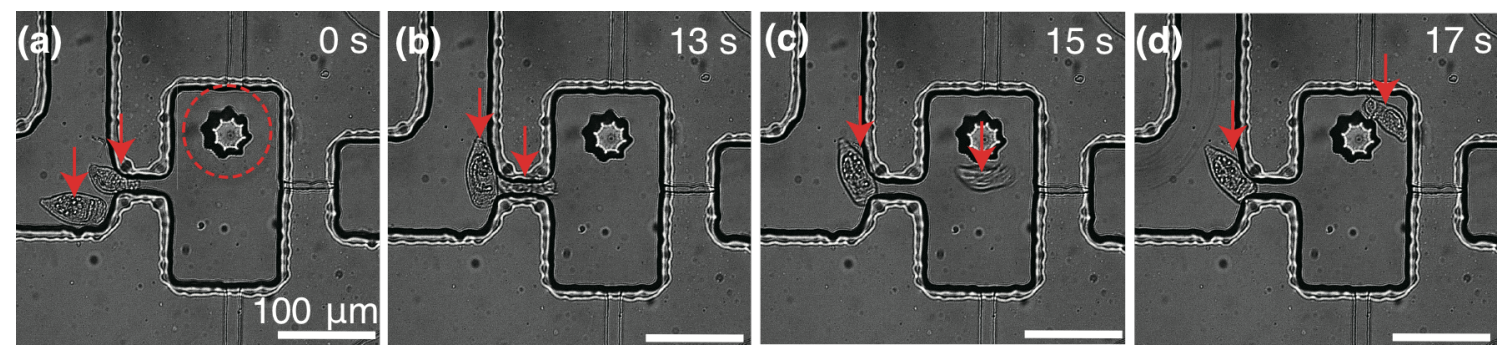

Fig. 5 Time series micrographs showing the procedure for trapping $V$. convallaria into a chamber. The chamber contained a polymerized gear structure (red dashed circle). A cell was pressed into the chamber by hydrodynamic pressure. Arrows indicate $V$. convallaria.
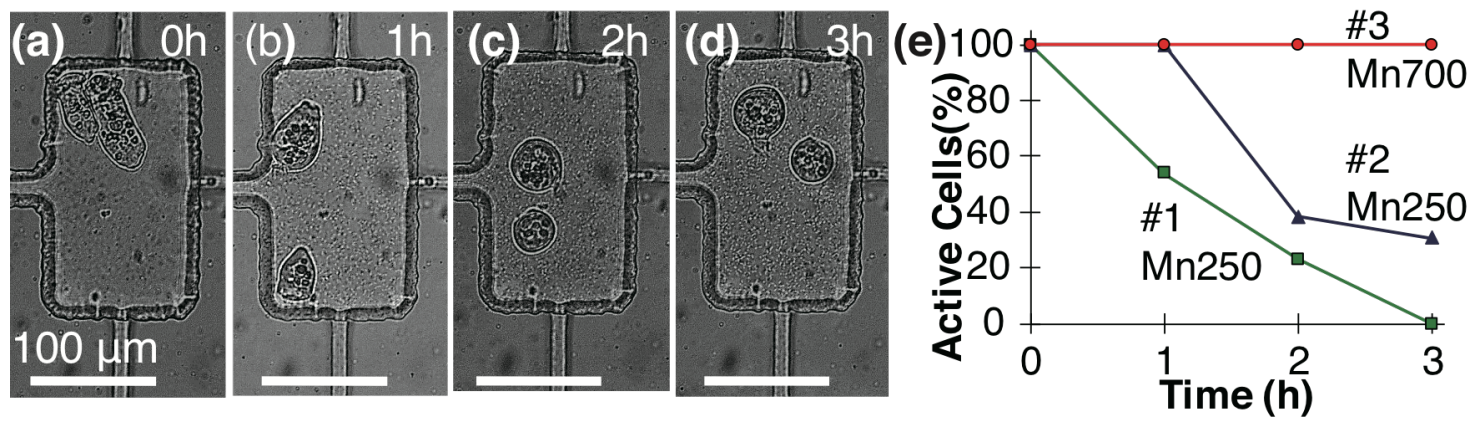

Fig. 6 Viability of $V$. convallaria in a microchamber. (a)-(d) Time series images of Vorticella inside a microchamber in condition \#2, without a PEG-DA structure. (e) Cell viability in conditions \#1-3. Sample sizes were $\mathrm{N}=13$ in \#1 and $\# 2$ and $\mathrm{N}=43$ in $\# 3$.

$(\mathrm{n}=5)$. A channel was filled with $5 \mathrm{wt} \%$ DMPA in PEG-DA (Mn 250). We scanned a microscope stage to sequentially polymerize a total of 14 PEG-DA hydrogels (Mn 250) onto a glass substrate in all 14 chambers. The microchamber containing the polymerized micro-structure was washed with DIW for $1 \mathrm{~h}$. The structures remained on the glass substrate.

We achieved introduction of a cell to a chamber, where a polymerized structure was formed (Fig. 5). A cell suspension at a concentration of $570 \mathrm{cells} / \mu \mathrm{L}$ was injected into the channel. The flow speed was adjusted to $150 \mu \mathrm{m} / \mathrm{s}$ by changing the hydrodynamic pressure. A cell reaching the entry site of a chamber was transported into the chamber. Flow moved the cell to the vicinity of the structure. The number of chambers trapping one or more cells was $6 / 14$. This flow transportation method is critical for achieving the second integration method.

The introduced cells were incubated in the chambers for $1 \mathrm{~h}$ to evaluate the activities of the cells. The cells in the chambers or bypass channels did not adhere to the substrate; therefore, they did not show any spontaneous motions. This result suggested that PEG-DA (Mn 250) or photoinitiators diffused into the PDMS channel and remained in PDMS. An increase in washing time with DIW or use of higher molecular weight PEG-DA is thought to decrease the amount of remaining molecules inside PDMS and improve cellular viability.

\subsection{Viability assay in a microchamber for examining second integration method}

We assessed the viability assay of $V$. convallaria in a microchamber after replacing PEG-DA with a culture medium (Fig. 6). To find conditions keeping $V$. convallaria active for longer time, we used PEG-DA (Mn 250 or 700) containing $5 \mathrm{wt} \%$ DMPA and compared the viability of $V$. convallaria in the following three conditions: (1) we used PEG-DA (Mn 250) to form a hydrogel; (2) we filled a channel with PEG-DA (Mn 250) and did not form a hydrogel. We skipped this formation process to compare \#1 and \#2 and evaluate the effect of the PEG-DA structure; (3) we used PEG-DA (Mn 700) to compare the effect of molecular weight. In all conditions \#1-3, channels were washed for $15 \mathrm{~h}$ with DIW prior to injecting V. convallaria. We set $15 \mathrm{~h}$ for cleaning, which was longer than $1 \mathrm{~h}$ in Section 3.3, to ensure further removal of remained molecules.

Cell viability was highest in condition \#3, which used PEG-DA (Mn 700). The viability at $3 \mathrm{~h}$ after $V$. convallaria injection was $100 \%(\mathrm{~N}=43)$, which was high enough for biohybrid system applications. Condition \#1 resulted in $0 \%$ cell activity $(\mathrm{N}=13)$ at $3 \mathrm{~h}$ after cell injection. Time series micrographs, collected during condition \#2, are shown in Figs. 6 (a)-(d). V. convallaria cell bodies became spherical at 1-2 $\mathrm{h}$ after injection. This cellular morphology alteration showed that $V$. convallaria was not active. Cellular activity was slightly better in condition $\# 2$, which lacked a structure, when compared to condition $\# 1$; the active cell rate was approximately $30 \%$ at $3 \mathrm{~h}$ after cell injection. The results from 
(a)

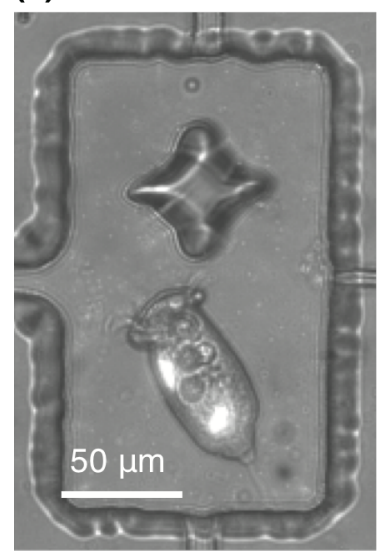

(b)

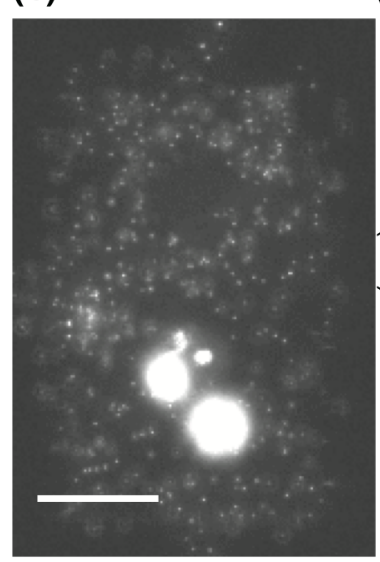

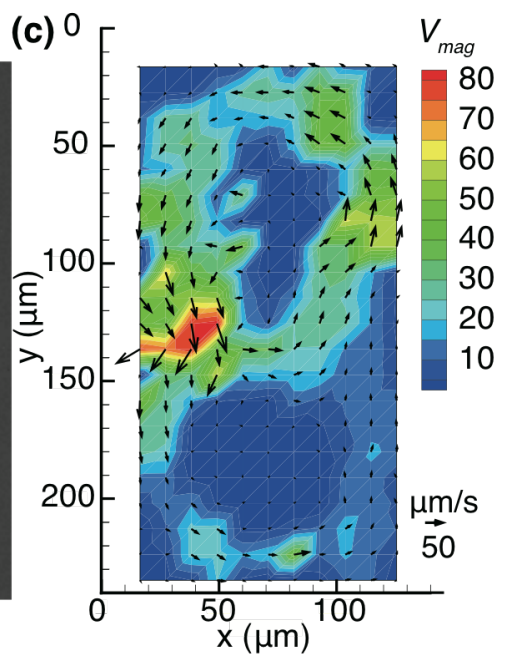

Fig. 7 Flow generation by a single V. convallaria cell in a chamber. (a) Bright field and (b) fluorescent images of Vorticella and fluorescent particles inside a chamber. (c) PIV analysis showing a flow field around the cell.

conditions \#1 and \#2 suggested that residual PEG-DA (Mn 250) affected the cells. The molecules remained in the PDMS device, even when the solution was washed for a longer time than described in Section 3.3.

We confirmed the effect of using higher molecular weight PEG-DA. The increase in PEG-DA molecular weight improved cellular activity. Possible explanations are less diffusion into PDMS and reduced toxicity to V. caonvallaria. The rationale for reduced toxicity is supported by similar experiments in mammalian cells, which showed that higher molecular weight PEG-DA resulted in higher cell viability compared to lower molecular weight PEG-DA (Shin, et al., 2003).

\subsection{Activity of Vorticella convarallia and flow measurement in chamber containing hydrogel structure}

We measured activity of a $V$. convallaria cell in a microchamber and demonstrated the second integration method. Higher molecular weight PEG-DA (Mn 700) with 5\% DMPA was used to form a PEG-DA hydrogel in a microchamber. We shortened the washing time with DIW to $5 \mathrm{~h}$ for reducing the total assembling time. $V$. convallaria cells were injected to chambers. 7 of 14 chambers were filled with $V$. convallaria. The injected cells adhered to a substrate at $1 \mathrm{~h}(5 / 7)$ and $2 \mathrm{~h}(5 / 7)$ after injection. $V$. convallaria escaped from 2 of the 7 chambers at $2 \mathrm{~h}$. The 5 remained cells were active and assembled contractile stalks (5/5). $5 \mathrm{~h}$ of washing time did not make much difference in the cell activities. Additional decrease in washing time is considered to be possible and can reduce the total production time.

Fluorescent particles were added to the chambers to visualize a flow generated by $V$. convallaria. The $V$. convallaria cells moved their cilia and particles were circulated in the chamber. We investigated a flow generated by 1 of the 5 samples using a PIV technique. Figure 7 shows a PIV analysis of a chamber containing a single cell of $V$. convallaria and a polymerized structure. Figure 7(a) shows $V$. convallaria adhering to a chamber surface and generating a flow inside the chamber. Figure 7(b) is a fluorescent microscope image, which shows fluorescent particles in the chamber. A PIV analysis is shown in Fig. 7 (c). The cell generated a counterclockwise flow around the structure. The boundary of the structure affected the flow. The fastest flow speed was $83 \mu \mathrm{m} / \mathrm{s}$ close to the mouth of $V$. convallaria.

We have found a way to keep V. convallaria cells active, when they are combined with PEG-DA hydrogels. The cilia motion indicated that $V$. convallaria was active around a PEG-DA structure. PEG-DA (Mn 700) was formed in a channel, then the channel was washed for $5 \mathrm{~h}$ prior to cell injection. One advantage of this method of cell placement after hydrogel formation was that toxic chemicals were removed as much as possible before injecting cells. Therefore, this method is better for maintaining a higher cell activity rate compared to the alternate method, where hydrogel formation occurs after cell placement.

\section{Conclusions}

In this study, we introduced microfluidic in situ photolithography techniques to biohybrid systems by fabricating a PEG-DA microstructure, which enables integration of $V$. convallaria into a PEG-DA hydrogel. We characterized the polymerized conditions of monomers and photoinitiators in a microchannel with the microscope-based optical setup. The 
reduction factors through the microscope were investigated by measuring the dimensions of fabricated structures.

To incorporate a PEG-DA structure with $V$. convallaria, we studied the two integration methods. The $V$. convallaria viability was assessed in two conditions to examine integration methods. The assessment of cellular viability in a PEGDA solution indicated that lower concentrations of PEG-DA and photoinitiator I-2959 improved cellular viability. This finding is useful for realization of 1) hydrogel formation after cell placement. We established 2) cell placement after hydrogel formation. A $V$. convallaria cell was trapped in a designed chamber containing a polymerized component by a microfluidic approach; the two components were combined. This microfluidic integration method of a preformed microstructure and a cell is applicable not only to $V$. convallaria but also to the different kinds of cells. The assay of cellular viability in a microchamber after exchanging the PEG-DA solution with culture medium showed that higher molecular weight PEG-DA was less toxic to $V$. convallaria. The activity of $V$. convarallia in a chamber containing a hydrogel structure showed the successful combination of $V$. convallaria and a PEG-DA hydrogel. A flow generated by $V$. convarallia was confirmed by PIV analysis. The cellular viability assessment revealed the potential use of $V$. convarallia as power sources for actuating a movable polymerized component.

We clarified the conditions to keep $V$. convallaria active, while simultaneously forming a hydrogel structure. This methodology introduces a movable component and new polymeric material for biohybrid systems. The microfluidic in situ photolithography techniques also allow rapid prototyping inside a microchannel. Those capabilities potentially achieve the dynamic state change of a microsystem and provide a wider range of biohybrid-system applications.

\section{Acknowledgements}

This work was supported by JSPS KAKENHI Grant Number JP15K13910, Hatakeyama Culture Foundation, and The Foundation for The Promotion of Ion Engineering.

\section{References}

Akiyama, Y., Sakuma, T., Funakoshi, K., Hoshino, T., Iwabuchi, K., and Morishima, K., Atmospheric-Operable Bioactuator Powered by Insect Muscle Packaged with Medium, Lab on a Chip, Vol.13, No.24 (2013), pp.4870-4880.

Carlsen, R. W., and Sitti, M., Bio-Hybrid Cell-Based Actuators for Microsystems, Small, Vol.10, No.19 (2014), pp.3831-3851.

Chung, S. E., Park, W., Shin, S., Lee, S. A., and Kwon, S., Guided and Fluidic Self-Assembly of Microstructures Using Railed Microfluidic Channels, Nat Mater, Vol.7, No.7 (2008), pp.581-587.

Dendukuri, D., Pregibon, D. C., Collins, J., Hatton, T. A., and Doyle, P. S., Continuous-Flow Lithography for High-Throughput Microparticle Synthesis, Nature materials, Vol.5, No.5 (2006), pp.365-369.

Hahn, M. S., Mchale, M. K., Wang, E., Schmedlen, R. H., and West, J. L., Physiologic Pulsatile Flow Bioreactor Conditioning of Poly(Ethylene Glycol)-Based Tissue Engineered Vascular Grafts, Annals of Biomedical Engineering, Vol.35, No.2 (2007), pp.190-200.

Koh, W.-G., Itle, L. J., and Pishko, M. V., Molding of Hydrogel Microstructures to Create Multiphenotype Cell Microarrays, Analytical Chemistry, Vol.75, No.21 (2003), pp.5783-5789.

Koh, W.-G., and Pishko, M. V., Fabrication of Cell-Containing Hydrogel Microstructures inside Microfluidic Devices That Can Be Used as Cell-Based Biosensors, Analytical and Bioanalytical Chemistry, Vol.385, No.8 (2006), pp.1389-1397.

Lu, L.-H., Ryu, K. S., and Liu, C., A Magnetic Microstirrer and Array for Microfluidic Mixing, Microelectromechanical Systems, Journal of, Vol.11, No.5 (2002), pp.462-469.

Madou, M., 2011, Fundamentals of Microfabrication: The Science of Miniturization, Taylor \& Francis Limited,

Mironi-Harpaz, I., Wang, D. Y., Venkatraman, S., and Seliktar, D., Photopolymerization of Cell-Encapsulating Hydrogels: Crosslinking Efficiency Versus Cytotoxicity, Acta Biomaterialia, Vol.8, No.5 (2012), pp.1838-1848.

Moeller, H.-C., Mian, M. K., Shrivastava, S., Chung, B. G., and Khademhosseini, A., A Microwell Array System for Stem Cell Culture, Biomaterials, Vol.29, No.6 (2008), pp.752-763.

Nagai, M., Hasayaka, Y., Kato, K., Kawashima, T., and Shibata, T., Mixing of Solutions by Coordinated Ciliary Motion in Vorticella convallaria and Patterning Method for Microfluidic Applications, Sensors and Actuators B: Chemical, (2013a),

Nagai, M., Matsumoto, N., Kawashima, T., and Shibata, T., Reversible Motion Control of Vorticella Stalk in Microchannel, Microelectronic Engineering, Vol.108, No.0 (2013b), pp.28-32. 
Nagai, M., Ryu, S., Thorsen, T., Matsudaira, P., and Fujita, H., Chemical Control of Vorticella Bioactuator Using Microfluidics, Lab on a Chip, Vol.10, No.12 (2010), pp.1574-1578.

Neužil, P., Giselbrecht, S., Länge, K., Huang, T. J., and Manz, A., Revisiting Lab-on-a-Chip Technology for Drug Discovery, Nat Rev Drug Discov, Vol.11, No.8 (2012), pp.620-632.

Oh, K. W., Lee, K., Ahn, B., and Furlani, E. P., Design of Pressure-Driven Microfluidic Networks Using Electric Circuit Analogy, Lab on a Chip, Vol.12, No.3 (2012), pp.515-545.

Revzin, A., Tompkins, R. G., and Toner, M., Surface Engineering with Poly(Ethylene Glycol) Photolithography to Create HighDensity Cell Arrays on Glass, Langmuir, Vol.19, No.23 (2003), pp.9855-9862.

Shin, H., Temenoff, J. S., and Mikos, A. G., In Vitro Cytotoxicity of Unsaturated Oligo[Poly(Ethylene Glycol) Fumarate] Macromers and Their Cross-Linked Hydrogels, Biomacromolecules, Vol.4, No.3 (2003), pp.552-560.

Tan, W.-H., and Takeuchi, S., A Trap-and-Release Integrated Microfluidic System for Dynamic Microarray Applications, Proceedings of the National Academy of Sciences, Vol.104, No.4 (2007), pp.1146-1151.

Toonder, J. D., Bos, F., Broer, D., Filippini, L., Gillies, M., De Goede, J., Mol, T., Reijme, M., Talen, W., Wilderbeek, H., Khatavkar, V., and Anderson, P., Artificial Cilia for Active Micro-Fluidic Mixing, Lab on a Chip, Vol.8, No.4 (2008), pp.533-541.

Vacchiano, E., Kut, J., Wyatt, M., and Buhse, H., A Novel Method for Mass-Culturing Vorticella, The Journal of protozoology, Vol.38, No.6 (1991), pp.609-613. 